\title{
Avaliação do impacto das abordagens bottom- up e top-down nas atividades da vida diária em pessoas com neglect - revisão sistemática
}

\author{
Evaluation of the impact of bottom-up and top- \\ down approaches on daily activities in people \\ with neglet - a systematic review
}

\author{
Marlene Neves Rosa' 1 \\ Andreia Amorim² \\ Ana Rita Martins ${ }^{4}$ (D) \\ Marisa Bartolo 3 (D) \\ Catarina Oliveira 5 (D) \\ Cândida G. Silva ${ }^{6}$ (1)

\begin{abstract}
'Autora para correspondência. Escola Superior de Saúde, Instituto Politécnico de Leiria (Leiria); ciTechCare - Center for Innovative Care and Health Technology (Leiria). Portugal. marlene.rosa@ipleiria.pt 2-5Escola Superior de Saúde, Instituto Politécnico de Leiria (Leiria). Portugal.5160252@my.ipleiria.pt, 5160223@my.ipleiria.pt,
\end{abstract} \\ 5160222@my.ipleiria.pt, 5160239@my.ipleiria.pt \\ ${ }^{6}$ Centro de Química de Coimbra, Universidade de Coimbra (Coimbra); Escola Superior de Saúde, Instituto Politécnico de Leiria (Leiria).
} Portugal. candida.silva@ipleiria.pt

RESUMO | INTRODUÇão: Atualmente não existe um consenso entre quais as caraterísticas das abordagens (bottom-up ou top-down) mais eficazes na reabilitação das Atividades da Vida Diária (AVDs) em pessoas com Síndrome de Neglect (SN). OBJETIVO: Caracterizar as abordagens (bottom-up ou top-down) e o seu impacto nas AVDs em adultos e idosos com SN. MÉTODOS: Esta revisão sistemática foi realizada de acordo com a recomendação PRISMA - Preferred Reporting Items for Systematic Reviews and Meta-Analyses. A pesquisa bibliográfica foi realizada nas bases de dados PubMed, Web of Science, PEDro e Cochrane. Foram considerados estudos experimentais em que pelo menos uma técnica das abordagens bottom-up e top-down fosse utilizada. A ferramenta Joanna Briggs Institute Critical Appraisal Checklist for RCTs foi utilizada para avaliar a qualidade metodológica dos estudos. Foi usada a seguinte combinação de palavras chave: Neglect Syndrome $O R$ Unilateral Syndrome OR Neglect (...) AND Activities of Daily Living OR Daily (...) AND Treatment $O R$ Intervention OR Technique (...). RESULTADOS: Foram incluídos 16 estudos, dos quais 9 incluem técnicas de abordagem bottom-up, 6 incluem técnicas de abordagem top-down e 1 artigo inclui técnicas das duas abordagens. As técnicas da abordagem bottom-up que aumentaram a independência nas AVDs foram Visuomotor Feedback Training, Smooth Pursuit Eye Movement Training e a combinação de Eye Patching com Constraint-induced Therapy. Na abordagem top-down as técnicas com os mesmos resultados foram Visual Scanning, Mental Practice, Continuous Theta Burst Stimulation e Transcranial Direct Current Stimulation. CONCLUSÃO: As abordagens bottom-up e top-down aumentam a independência nas AVDs e cada uma contém técnicas com significativo impacto positivo, como Visuomotor Feedback Training e Continuous Theta Burst Stimulation. O presente trabalho permitiu uma análise crítica à classificação das abordagens em bottom-up e top-down, uma vez que não são sensíveis à distinção dos mecanismos de reabilitação envolvidos.

PALAVRAS-CHAVE: Autonegligência. Transtornos de Perceção. Atividades quotidianas. Reabilitação.
ABSTRACT | BACKGROUND: Currently there is no consensus on which are the characteristics of rehabilitation approaches (bottom-up or top-down) most effective in the rehabilitation of ADLs in people with Neglet Syndrome (NS). AIM: To characterize the approaches (bottomup or top-down) with more impact on ADLs in adults and elderly with NS. METHODS: This systematic review was carried out in accordance with the Preferred Reporting Items for Systematic Reviews and Metaanalyses (PRISMA) recommendation. A bibliographic search was carried out in PubMed, Web of Science, PEDro and Cochrane databases. Experimental studies were considered in which at least one technique of the bottom-up and top-down approaches was used. The Joanna Briggs Institute Critical Appraisal Checklist for RCTs tool was used to assess the methodological quality of the studies. The following combination of keywords was used: Negligence Syndrome OR Unilateral Syndrome OR Negligence (...) AND Activities OF Daily OR Daily Life (...) AND Treatment OR Intervention OR Technique (...). RESULTS: 16 studies were included, which 9 include techniques from the bottom-up approach, 6 include techniques from the top-down approach and 1 article includes two techniques, each belonging to each approach. The techniques of the bottom-up approach that increased independence in the ADLs were Visuomotor Feedback Training, Smooth Pursuit Eye Movement Training and the combination of Eye Patching with Constraint-induced Therapy. In the top-down approach, the techniques with the same results were Visual Scanning, Mental Practice, Continuous Theta Burst Stimulation and Transcranial Direct Current Stimulation. CONCLUSION: Both bottom-up and top-down approaches increase independence in ADLs. Each approach contains techniques with a significant positive impact on ADLs such as Visuomotor Feedback Training and Continuous Theta Burst Stimulation. The present work also allowed a critical analysis to the classification of the approaches in bottom-up and top-down, since they are not different to the category of rehabilitation mechanisms.

KEYWORDS: Self-Neglect. Perceptual Disorders. Activities of daily living. Rehabilitation. 


\section{Introdução}

A Síndrome de Neglect (SN) é uma característica incapacitante que provém de um desequilíbrio na excitabilidade inter-hemisférica ${ }^{1}$ com origem sobretudo no Acidente Vascular Cerebral (AVC). Ocorre em aproximadamente $50 \%$ dos seus sobreviventes e é mais grave e prevalente no hemisfério direito, com uma incidência de $13 \%$ a $82 \%$ 2 . Quanto aos seus sintomas, $20 \%$ a $80 \%$ dos mesmos aparecem na fase aguda e persistem em $75 \%$ dos indivíduos na fase crónica ${ }^{3,4}$. Apesar desta condição ser heterogénea, a maior parte dos indivíduos não responde a estímulos localizados no espaço contralesional, comprometendo a realização das Atividades da Vida Diária (AVDs) ${ }^{4,5}$.

A reabilitação da $\mathrm{SN}$ induz os indivíduos a explorarem o seu espaço negligenciado, sendo que as abordagens utilizadas podem ser divididas em bottom-up ou top-down $n^{2}$. A abordagem bottom-up tem como base a manipulação do ambiente sensorial privilegiando a estimulação externa como forma de aumentar a ativação do hemisfério lesado e o lado do corpo negligenciado ${ }^{6}$. Como exemplos, esta abordagem inclui as técnicas Prism Adaptation, Constraint-induced Therapy e Eye Patching. Por sua vez, a abordagem top-down recorre a aprendizagens previamente adquiridas para influenciar a perceção, necessitando de elevados níveis de atenção e colaboração por parte do indivíduo e requerendo algum grau de consciência da sua negligência ${ }^{-6-8}$. Como exemplos, esta abordagem engloba as técnicas Visual Scanning, Mental Practice e Continuous Theta Burst Stimulation.

Sobre a temática em estudo, uma revisão sistemática foi conduzida com o objetivo de determinar a eficácia de intervenções baseadas no treino de atividades funcionais e intervenções não baseadas em atividades (ex., eletroestimulação) na melhoria do desempenho funcional das AVDs e na redução de sintomas da negligência ${ }^{3}$. Esta revisão concluiu que técnicas como TENS, estimulação somatosensorial ou terapia de espelho são eficazes na redução dos sintomas no SN. Contudo, a maioria dos estudos incluídos não considerou a transferências destes resultados para a independência nas $\mathrm{AVDs}^{3}$. Uma outra revisão da literatura conduzida em 2017, cujo objetivo foi a descrição da eficácia das abordagens bottom-up e top-down na reabilitação para a SN , revelou a mesma limitação metodológica, por não explorar a transferência dos resultados obti-

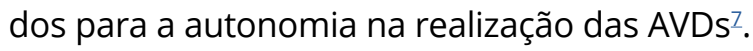

Considerando as dúvidas existentes nestes trabalhos prévios de revisão sistemática, revisitar e caracterizar a literatura sobre a eficácia das abordagens bottomup e top-down nas AVDs ${ }^{4}$ é crucial para facilitar o processo de decisão clínica na reabilitação da SN. Assim o presente trabalho tem como objetivo caracterizar as abordagens (bottom-up ou top-down) com maior impacto nas AVDs em adultos e idosos com SN.

\section{Metodologia}

Esta revisão sistemática foi realizada de acordo com a recomendação PRISMA Preferred Reporting Items for Systematic Reviews and Meta-Analyses (registro PROSPERO CRD42020201670) ${ }^{10}$.

\section{Critérios de inclusão e exclusão}

Foram incluídos estudos controlados randomizados (RCTs) em que técnicas das abordagens bottom-up e top-down foram aplicadas a pessoas com idade igual ou superior a 18 anos e com síndrome de Neglect (SN) e nos quais foram utilizados instrumentos para a avaliação das limitações nas AVDs com indicação do score antes e depois da intervenção. Foram considerados todos os estudos publicados até abril de 2020, disponíveis em texto integral e escritos em inglês, português ou espanhol. Foram excluídos estudos publicados em idiomas diferentes de inglês, português ou espanhol; estudos que recorram a intervenções farmacológicas; estudos sem diferenciação de participantes com e sem SN; estudos que incluam técnicas classificadas como abordagem mista (bottom-up e top-down); estudos cujo texto integral não se encontrava disponível; e estudos que não estejam concluídos. 


\section{Estratégia de pesquisa}

A pesquisa bibliográfica foi realizada nas bases de dados PubMed, Web of Science, PEDro e Cochrane, utilizando a seguinte estratégia de pesquisa (Neglect Syndrome $O R$ Unilateral Syndrome $O R$ Neglect $O R$ Hemineglect OR Spatial Neglect) AND (Activities of Daily Living OR Daily Routine OR Daily Living) AND (Treatment $O R$ Intervention $O R$ Technique OR Rehabilitation $O R$ Program).

\section{Seleção dos estudos e extração dos dados}

Numa primeira etapa, os estudos foram selecionados com base no título e no resumo; na segunda etapa foi lido o texto integral dos estudos. Estas duas etapas foram realizadas por dois avaliadores independentes. O coeficiente Kappa de Cohen foi calculado para avaliar a concordância entre investigadores na fase de seleção de estudos com base no título e resumo, e na leitura do texto integral. As divergências foram resolvidas por discussão e reunião de consenso. Os valores obtidos foram interpretados de acordo com os seguintes critérios: [0; 0,2] concordância fraca, [0,21; $0,4]$ concordância razoável, $[0,41 ; 0,6]$ concordância moderada, $[0,61 ; 0,8]$ concordância forte, $[0,81 ; 1]$ concordância quase perfeita. Estes cálculos foram realizados com o programa IBM SPSS versão 26.

Os dados foram extraídos para uma tabela com os seguintes dados: citação (último nome do primeiro autor e ano), características do grupo de intervenção (GI) e grupo controlo (GC) (número de participantes, idade, estadio da SN), parâmetros dos programas de reabilitação aplicados ao Gl e GC (técnica, número de sessões diárias, número de sessões semanais e número de semanas), instrumentos utilizados para a avaliação das limitações nas AVDs com indicação do score antes e depois da intervenção no GI e GC, principais conclusões.

\section{Risco de viés nos estudos}

O risco de viés dos estudos selecionados foi avaliado por dois investigadores independentes recorrendo à ferramenta Joanna Briggs Institute Critical Appraisal Checklist for RCTs ${ }^{11}$. Esta é constituída por 13 questões e permite a avaliação e determinação da possibilidade de viés no desenho e realização dos estudos (Questões 1 a 8) e na análise dos dados (Questões 9-13). Estas podem ser respondidas com os valores Yes, No, Unclear ou Not Applicable. Os intervalos de valores para a qualidade metodológica definidos foram como: muito pobre (0-2), pobre (3-5), moderada (6-8), elevada (9-11) e excelente (12-13) ${ }^{11}$.

\section{Resultados}

\section{Seleção dos estudos}

Após a pesquisa na literatura obtiveram-se 126 artigos, pelo que com a remoção dos duplicados sobraram 96 artigos, dos quais foram excluídos 58 por não abordarem a $\mathrm{SN}$ e/ou não referirem a influência da reabilitação nas AVDs (com base na leitura do título e resumo). Aos 38 artigos restantes e após a leitura do texto integral excluíram-se outros 22 de acordo com os critérios de exclusão definidos, obtendo-se um total de 16 estudos para síntese qualitativa (Figura 1). De acordo com os critérios de elegibilidade definidos, os estudos foram excluídos porque (i) o artigo não estava escrito em inglês, português ou espanhol $(n=3)$, (ii) o texto integral não se encontrava disponível $(n=5)$, (iii) o estudo não estava concluído $(n=6)$, (iv) não foi aplicado um instrumento para a avaliação das limitações nas AVDs $(n=2)$, ( $v$ ) a intervenção tinha uma abordagem mista (top-down e bottom-up) $(n=1)$, e (vi) não era feita uma diferenciação dos participantes $(n=5)$. Os valores Kappa de Cohen obtidos foram de 0,68 na seleção dos estudos com base no título e no resumo (concordância forte) e de 0,95 na seleção dos estudos tendo em conta a leitura do texto integral (concordância quase perfeita) ${ }^{12}$. 


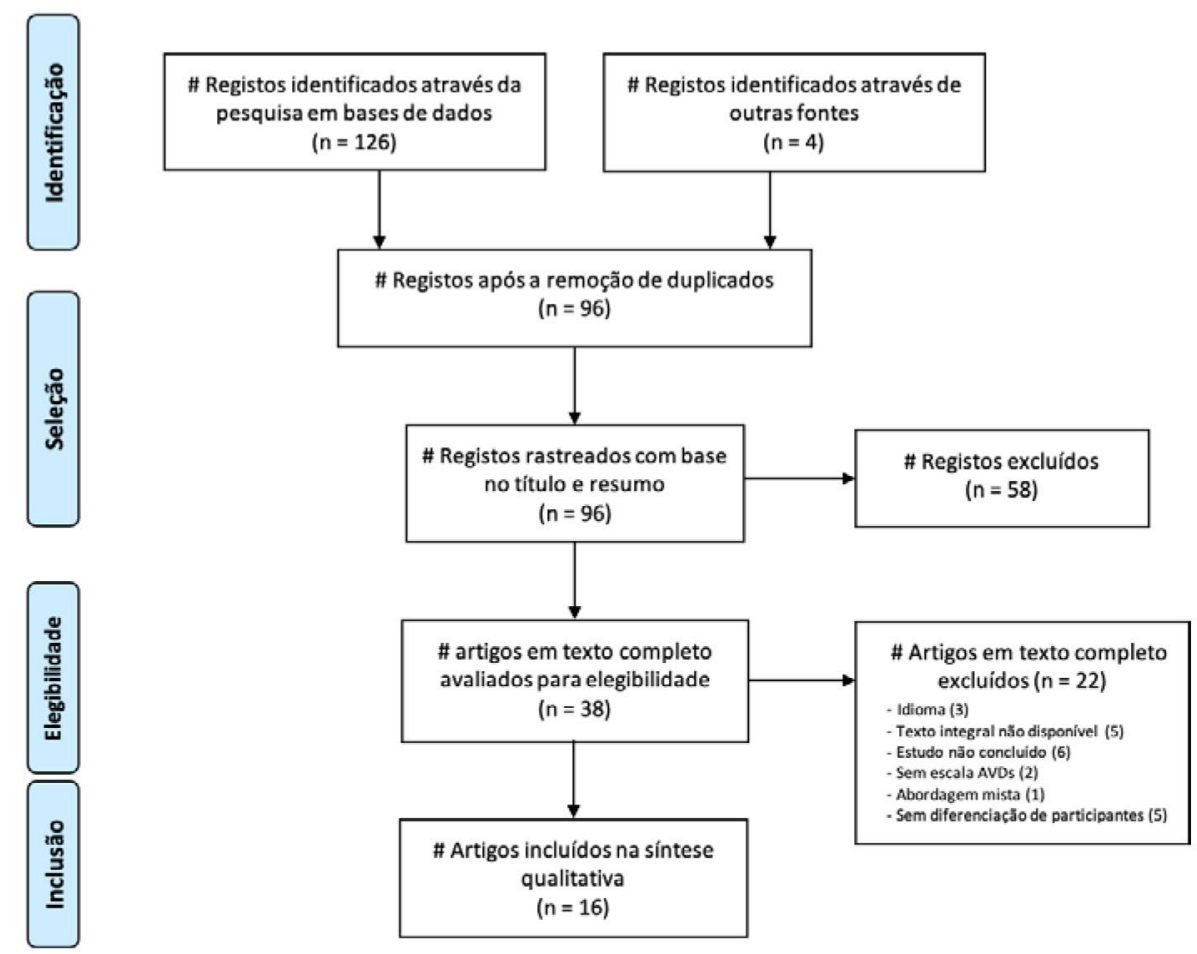

\section{Estudos com Abordagem bottom-up}

Dos 16 estudos incluídos, 9 estudos aplicaram intervenções com técnicas bottom-up (Quadro 1.). Nestes estudos foram incluídos 346 participantes, dos quais 194 pertenceram ao grupo de intervenção e 152 ao grupo de controlo. Dois estudos 13,14 , incluíram dois grupos de intervenção. A maioria dos estudos apresentou a média das idades que variou no grupo de intervenção de 56,1 a 74,21 anos e no grupo de controlo de 61,33 a 73,8 anos. Quanto ao estadio da SN, 2 estudos ${ }^{15,16}$ intervieram na fase aguda, 6 estudos ${ }^{13,17-21}$ na fase subaguda e 1 estudo 22 na fase crónica, sendo que 1 dos estudos ${ }^{14}$ não referiu a fase em que interveio.

Foram utilizado 5 instrumentos diferentes para a avaliar as limitações dos participantes nas AVDs antes e após a intervenção: Catherine Bergego Scale (CBS) [5 estudos $14,15,18-20]$, Barthel Index (BI) [3 estudos ${ }^{15,16,21}$ ], Functional Independence Measure (FIM) [3 estudos $^{13,17,19}$ ], Score of Independence Index for Neurological and Geriatric Rehabilitation (SINGER) [1 estudo ${ }^{21}$ ] e Stroke Impact Scale (SIS) [1 estudo2] ]. Além disso, 4 estudos ${ }^{13,17-19}$ aplicaram o Behavioural Inattention Test (BIT) para avaliar a gravidade da SN.

As técnicas bottom-up utilizadas foram Prism Adaptation [3 estudos ${ }^{18-20}$ ], Eye Patching [4 estudos $^{13-15,17}$ ], robótica [1 estudo ${ }^{21}$ ], Visuomotor Feedback Training [1 estudo ${ }^{22}$ ] e Smooth Pursuit Eye Movement Training [1 estudo $\left.{ }^{16}\right]$.

Os estudos que utilizaram a técnica Prism Adaptation no grupo de intervenção ${ }^{18-20}$, compararam a técnica com placebo (GC). Apenas Mizuno et al. (2011) ${ }^{19}$ concluiu que o grupo de intervenção obteve resultados mais significativos que o controlo na independência nas AVDs (Scores da CBS Pré-intervenção: $\mathrm{Gl}=9.8 \pm 6.8 ; \mathrm{GC}=9.6 \pm 6.2$ / Pós-intervenção: $\mathrm{Gl}=4.8 \pm 3.7$; $\mathrm{GC}=6.4 \pm 5.1$ ) (Tabela 1). Adicionalmente, Turton et al. $(2010)^{18}$ concluiu que a mesma técnica apresentou resultados mais significativos na diminuição dos sintomas da SN subaguda quando comparada ao placebo (Scores do BIT-C Pré-intervenção: $\mathrm{Gl}=88 \pm 71$; $\mathrm{GC}=109 \pm 70$ / Pós-intervenção: Gl=variação positi-

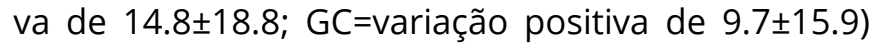
(Quadro 1). 
Nos estudos incluídos, a técnica Eye Patching foi sempre aplicada em combinação com outras técnicas. Em Tsang et al. (2009)17, a combinação da técnica Eye Patching com Terapia Ocupacional Convencional (Gl) foi comparada com Terapia Convencional. Fong et al. (2007) ${ }^{13}$ combinaram as técnicas Eye Patching e Voluntary Trunk Rotation Training (GI1) comparando com Voluntary Trunk Rotation Training (GI2) e com Terapia Ocupacional Convencional. Wu et al. (2013) ${ }^{14}$ associaram as técnicas Eye Patching e ConstraintInduced Therapy (GI1) sendo realizada a comparação com a aplicação de Constraint-Induced Therapy apenas (GI2) e com Terapia Ocupacional (GC). Por seu lado, Machner et al. (2014) $)^{15}$ associaram as técnicas Eye Patching e Optokinetic Stimulation (GI) e compararam-nas com um tratamento não específico para a SN (GC). Apenas Wu et al. (2013) 14 inferiu que a técnica Eye Patching associada a Constraint-Induced Therapy (GI1) se traduziu em melhorias significativas na independência nas AVDs quando comparada a ConstraintInduced Therapy isolada (GI2) ou Terapia Ocupacional Convencional (GC) (Scores da CBS Pré-intervenção: $\mathrm{GI}=16.1 \pm 3.2 ; \quad \mathrm{GI}=13.9 \pm 4.8 ; \quad \mathrm{GC}=18.1 \pm 5.1 /$ Pósintervenção: $\mathrm{Gl} 1=10.4 \pm 3.2 ; \mathrm{Gl}=9.9 \pm 4.4 ; \mathrm{GC}=16.3 \pm 4.5$ ) (Tabela 1).

Para a intervenção robótica, Karner et al. (2019) ${ }^{21}$ comparou a utilização do robot PARO (Gl), que estimula uma relação com os participantes através do toque, com a leitura de um livro em voz alta (GC) e concluiu que a aplicação do robot não levou a resultados significativos na independência nas AVDs (Scores da
SINGER Pré-intervenção: $\mathrm{GI}=7.95 \pm 4.63 ; \mathrm{GC}=8.22 \pm 4.49$ / Pós intervenção: $G \mid=12.48 \pm 5.44 ; G C=11.11 \pm 5.41$ ) (Tabela 1).

Rossit et al. (2019)22 concluiu que a técnica Visuomotor Feedback Training (Gl) é um método de reabilitação eficaz e pode ser realizada em casos de SN crónica, para além de apresentar melhorias nas AVDs quando comparada à intervenção do grupo de contro-

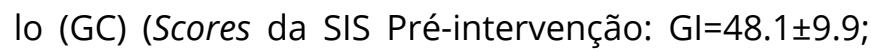
$\mathrm{GC}=46.0 \pm 8.7$ / Pós-intervenção: $\mathrm{Gl}=50.1 \pm 9.5$; $\mathrm{GC}=56.7 \pm 7.0$ ) (Tabela 1).

Para a técnica Smooth Pursuit Eye Movement Training, Kerkhoff et al. (2014) $)^{16}$ comparou a sua aplicação com a técnica Visual Scanning (abordagem topdown) e concluiu que a primeira (GI1) revelou melhorias mais significativas na redução dos sintomas da SN aguda que Visual Scanning (GI2), apesar de ambas aumentarem a independência nas AVDs (Scores do BI Pré-intervenção: $G \mid 1=11 \pm 4 ; G I 2=15 \pm 5$ / Pós intervenção: $\mathrm{Gl} 1=28 \pm 5 ; \mathrm{Gl} 2=26 \pm 8$ ) (Tabela 1).

No que diz respeito à dose de intervenção, esta variou de 1 a 2 sessões diárias, 2 a 7 dias por semana ao longo de 1 a 6 semanas. No que se refere ao follow-up, 5 estudos ${ }^{14,17,18,20,21}$ realizaram-no de forma incompleta: em 2 estudos ${ }^{14,17}$ o follow-up não foi realizado e em 3 estudos $^{18,20,21}$ não foi especificado o motivo da desistência de alguns participantes. 


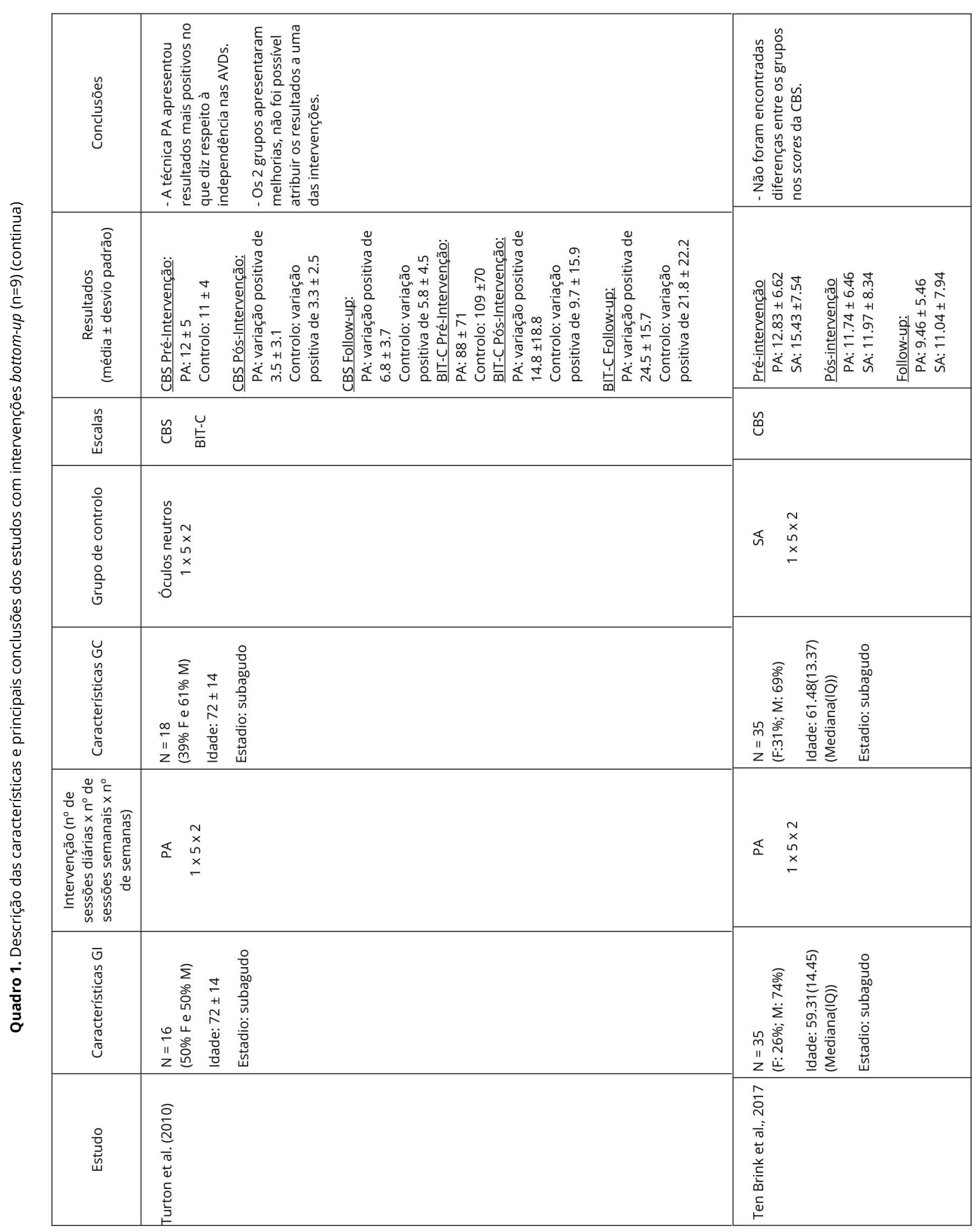




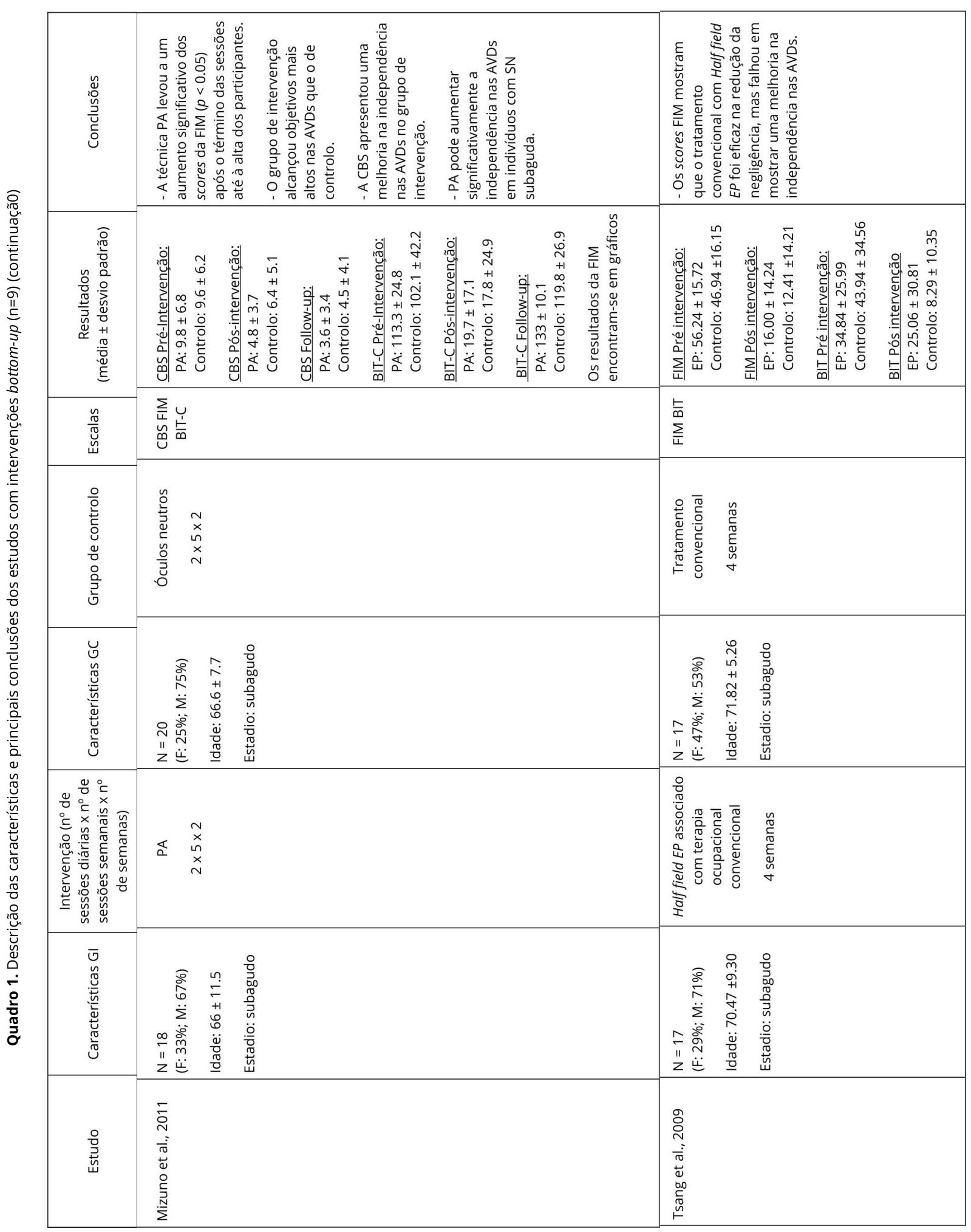




\begin{tabular}{|c|c|}
\hline 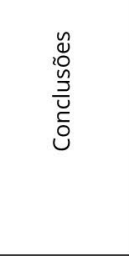 & 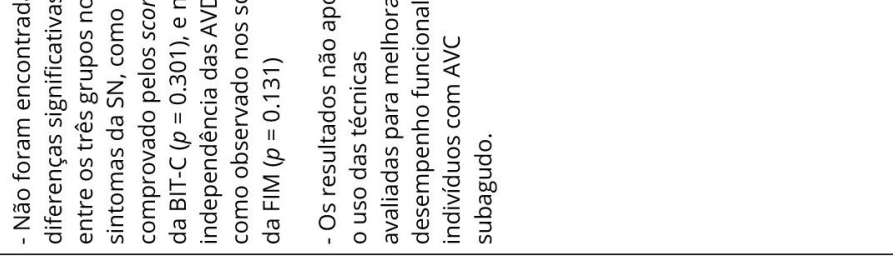 \\
\hline 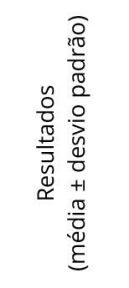 & 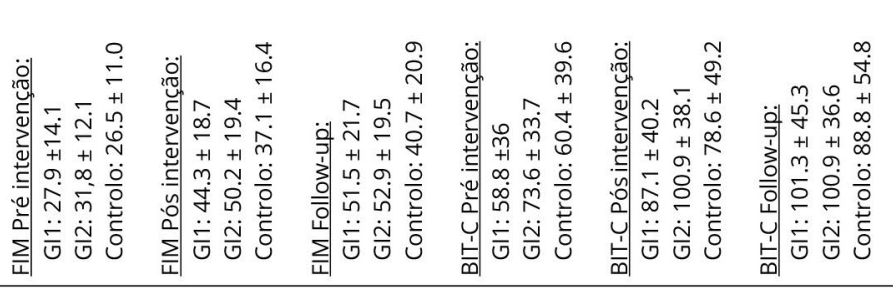 \\
\hline 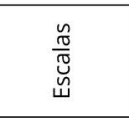 & 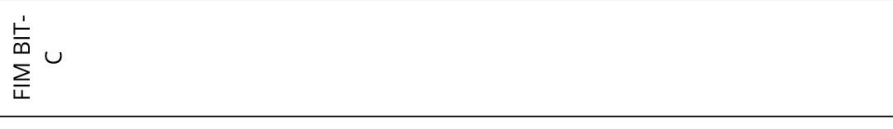 \\
\hline 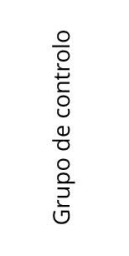 & 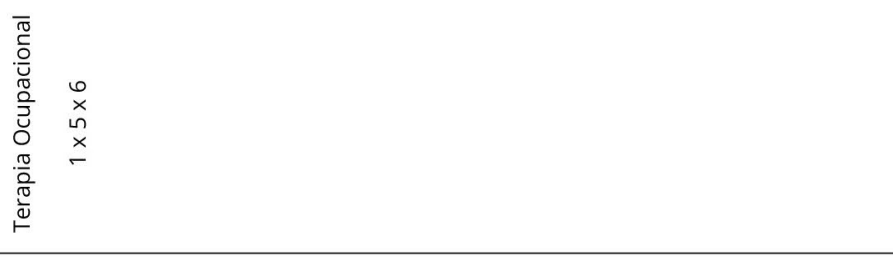 \\
\hline 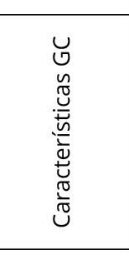 & 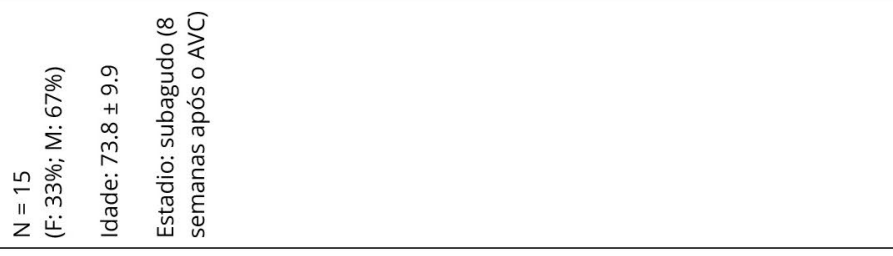 \\
\hline 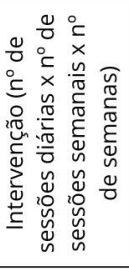 & 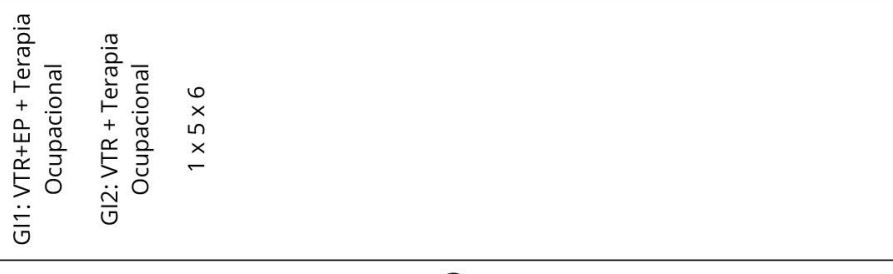 \\
\hline 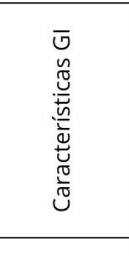 & 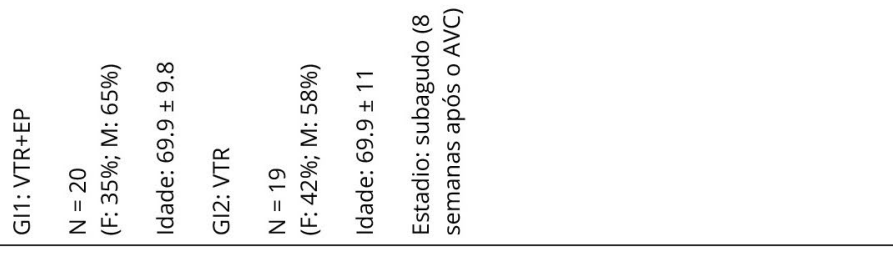 \\
\hline 总 & 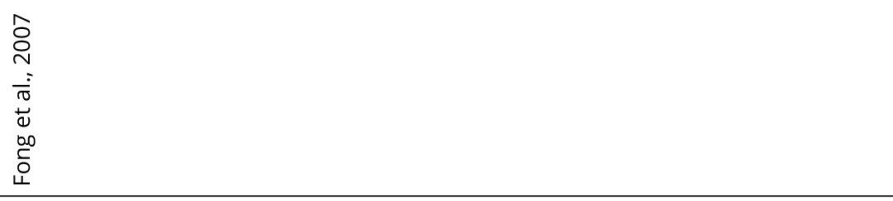 \\
\hline
\end{tabular}




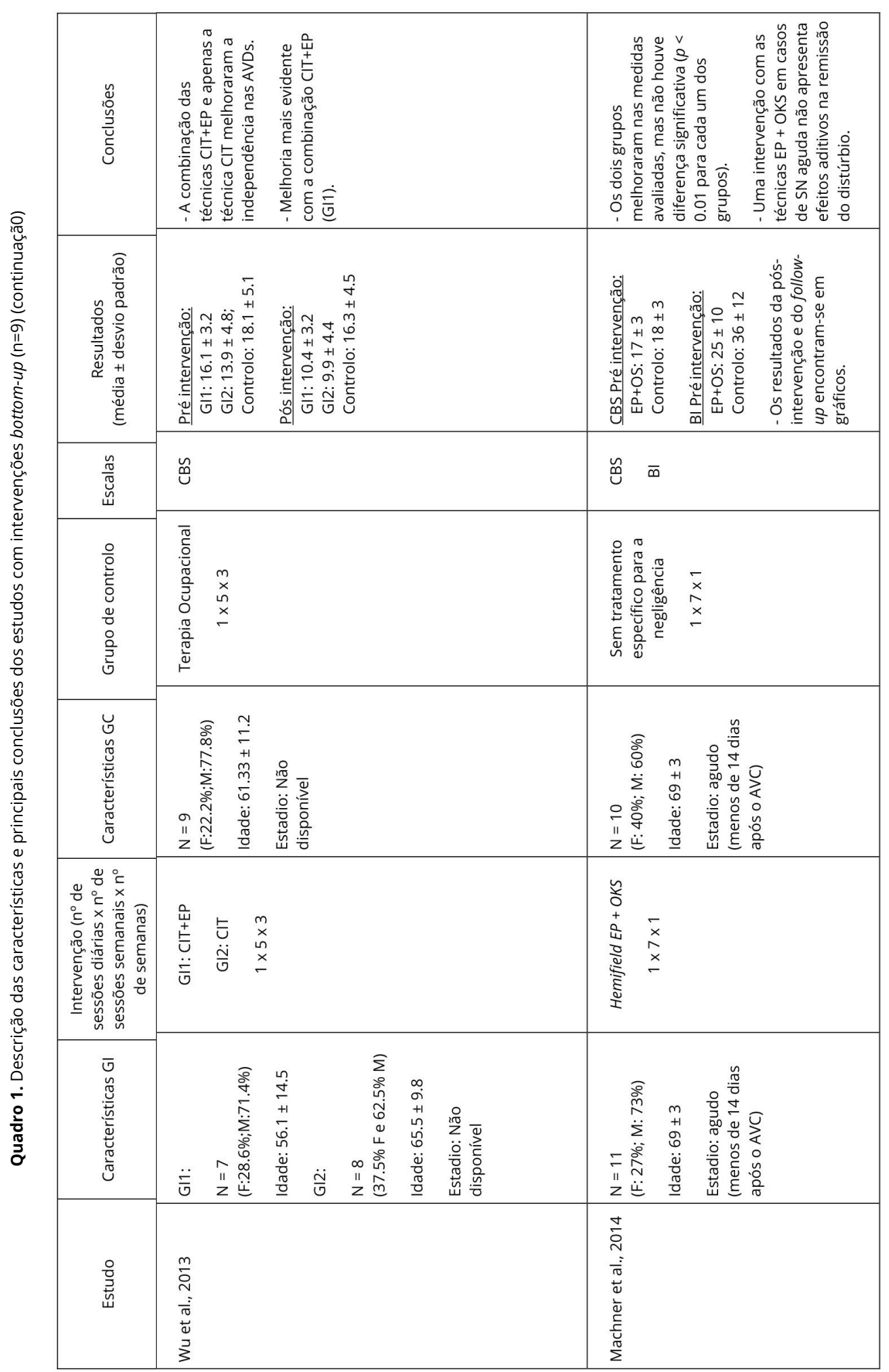




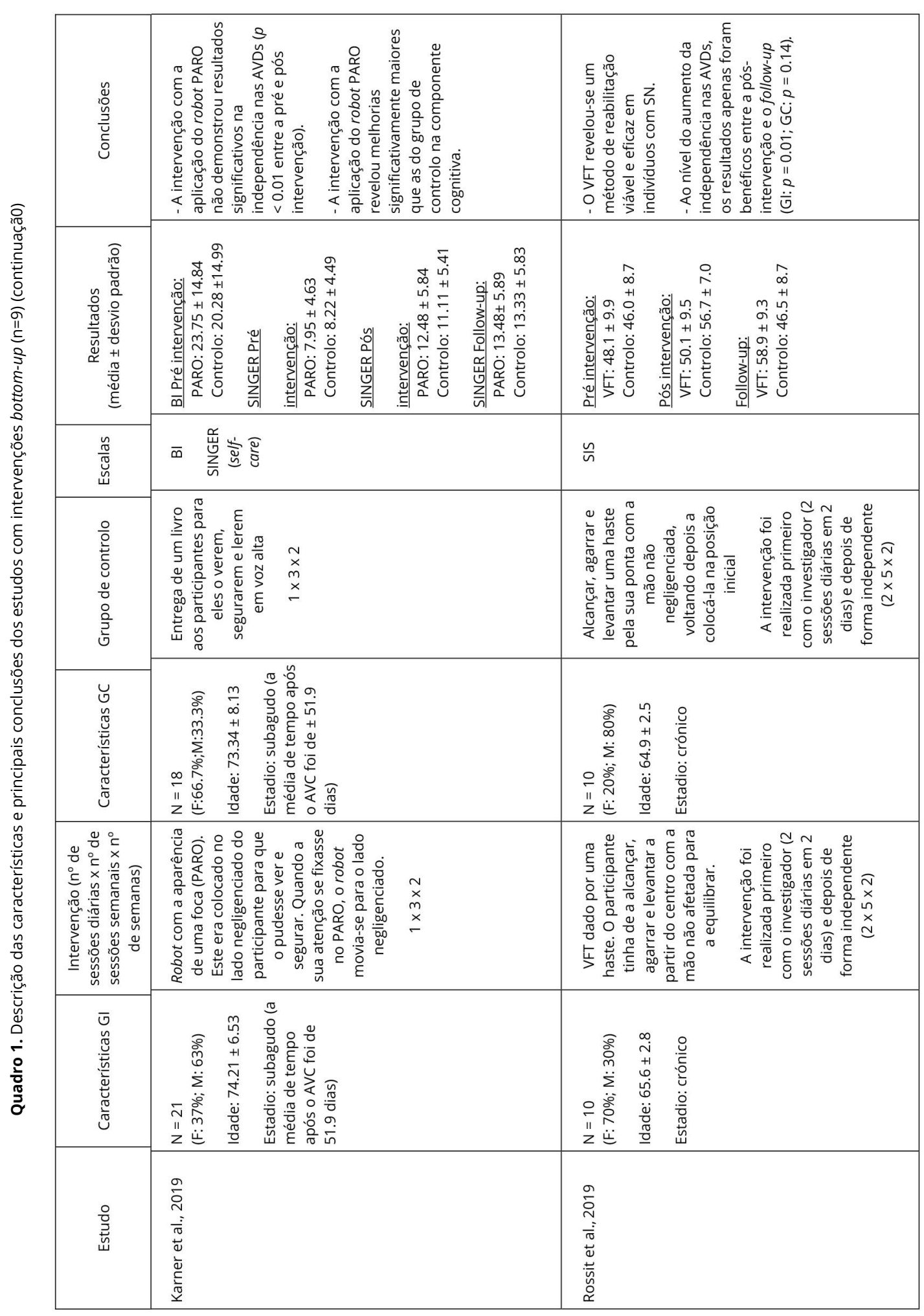




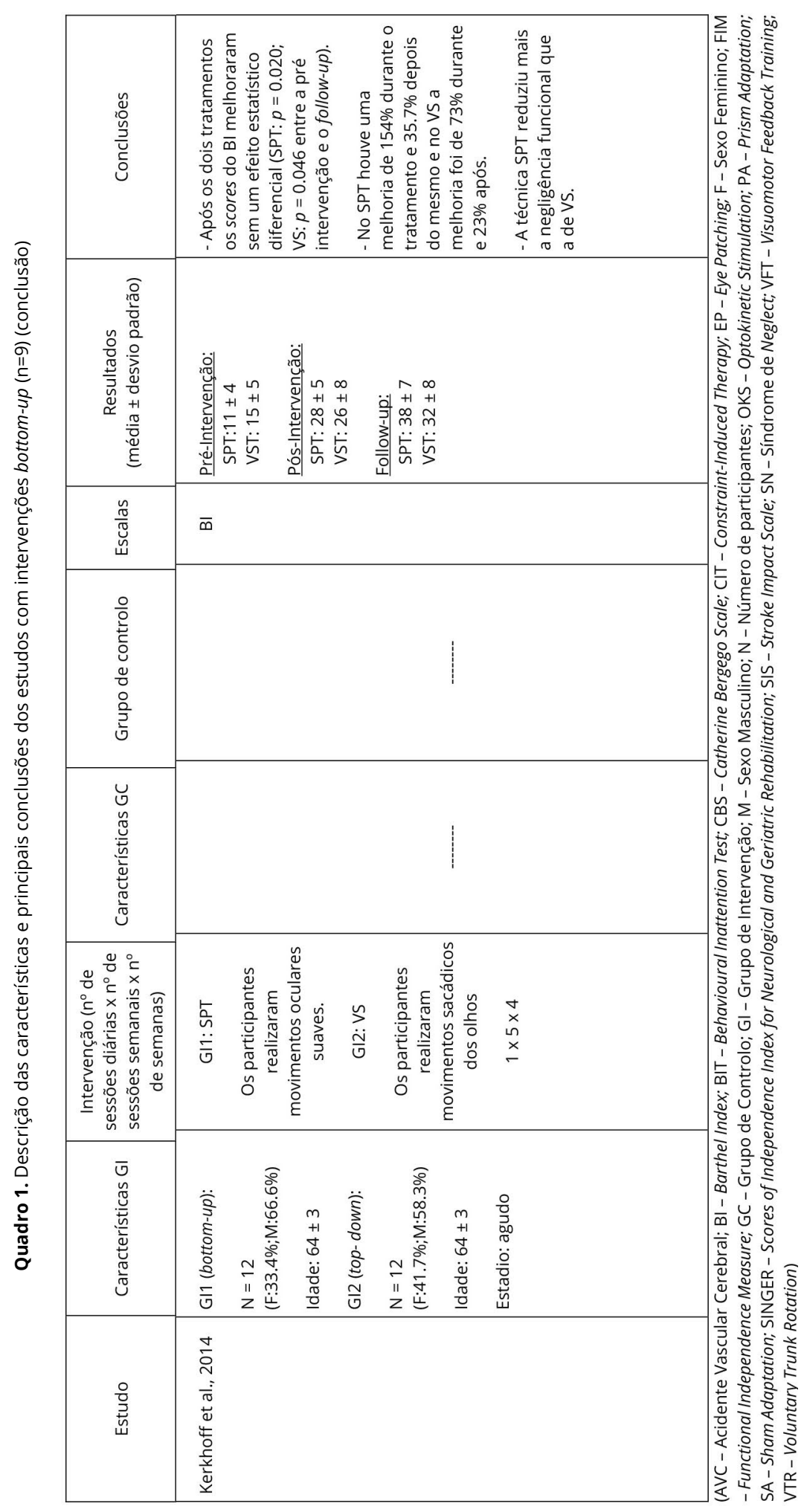




\section{Estudos com Abordagem top-down}

As intervenções com técnicas top-down foram descritas em 7 estudos (Quadro 2). Nestes estudos participaram 207 participantes, dos quais 126 pertenceram ao grupo de intervenção e 57 ao grupo de controlo. Num dos estudos ${ }^{23}$, estiveram envolvidos 24 participantes mas não é indicado o número de participantes em cada grupo. A maioria dos estudos apresentou a média das idades dos participantes, que variou entre 54,6 e 74,3 anos no grupo de intervenção, e entre 58,7 e 70,6 anos no grupo de controlo. Quanto ao estadio da SN, 2 estudos $\frac{16,24}{1}$ intervieram na fase aguda, 4 estudos $1,23,25,26$ na fase subaguda e 1 estudo ${ }^{27}$ nas três fases.

Foram utilizados 4 instrumentos diferentes para a avaliar as limitações dos participantes nas AVDs antes e após a intervenção: CBS [4 estudos ${ }^{1,23,25,26}$ ], BI [3 estudos $^{16,24,25}$ ] e FIM [2 estudos ${ }^{26,27}$ ]. Apenas 2 estudos ${ }^{1,27}$ recorreram ao BIT para avaliar a gravidade da SN.

As técnicas top-down utilizadas foram Visual Scanning [3 estudos $16,24,27]$, Mental Practice [1 estu$\mathrm{do}^{25}$ ], Continuous Theta Burst Stimulation [2 estudos $^{23,26}$ ], Transcranial Direct Current Stimulation [1 estudo ${ }^{25}$ ], e Repetitive Transcranial Magnetic Stimulation [1 estudo'].

Van Wyk et al. (2014)24 comparou a técnica Visual Scanning associada a Saccadic Eye Movement Training integrada às atividades específicas da tarefa (GI) com um treino de atividades específicas da tarefa apenas (GC) e concluiu que a combinação de técnicas revelou um efeito significativo no aumento da independência nas AVDs. Por sua vez, Ferreira et al. (2011) 27 verificou que a técnica Visual Scanning (GI1) obteve resultados mais significativos que a técnica Mental Practice (GI2) visto ter melhorado os sintomas da SN subaguda, aguda e crónica e aumentado a independência nas AVDs (Scores da FIM sob a forma de mediana, min/ max na Pré-intervenção: $G \mid 1=81,41 / 117 ; G I 2=76$, 62/120 / Pós-intervenção: GI1=84, 60/121; GI2= 79, 69/125). Kerkhoff et al. (2014) ${ }^{16}$, como anteriormente mencionado, concluiu que a técnica Smooth Pursuit Eye Movement Training revelou melhorias mais significativas na redução dos sintomas da SN aguda que Visual Scanning (Quadro 2).
Nyffeler et al. (2019) ${ }^{26}$ comparou dois métodos da técnica Continuous Theta Burst Stimulation com placebo e concluiu que esses dois protocolos reduziram a gravidade da SN subaguda e obtiveram um impacto positivo nas AVDs. Por sua vez, Cazzoli et al. (2012) ${ }^{23}$ comparou três grupos: aplicação da técnica Continuous Theta Burst Stimulation seguida de placebo (GI1), placebo seguido de Continuous Theta Burst Stimulation (GI2) e placebo (GC). Este estudo concluiu que a técnica Continuous Theta Burst Stimulation produziu uma melhoria substancial na SN subaguda e na independência nas AVDs (Tabela 2).

Para a técnica Transcranial Direct Current Stimulation, Yi et al. (2016) $\frac{25}{}$ realizou uma comparação entre esta técnica na forma anodal (GI1), a mesma técnica na forma cathodal (GI2) e placebo (GC), tendo concluído que as duas primeiras técnicas combinadas com Terapia Ocupacional e Fisioterapia apresentaram melhorias nos sintomas da SN subaguda. No entanto, essas melhorias não se repercutiram nas AVDs (Scores da CBS Pré-intervenção: GI1 $=17 \pm 10.6$; $\mathrm{GI}=16.2 \pm 6.4 ; \quad \mathrm{GC}=16.0 \pm 9.7$ / Pós-intervenção: $\mathrm{Gl}=8.4 \pm 9 ; \mathrm{Gl}=10 \pm 6.2 ; \mathrm{GC}=12.3 \pm 10.8$ ) (Tabela 2).

Por fim, para a técnica Repetitive Transcranial Magnetic Stimulation, Yang et al. (2017) 1 associou-a à técnica Sensory Cueing (GI1) e comparou essa combinação com Repetitive Transcranial Magnetic Stimulation apenas (GI2) e com Terapia Convencional (GC). Dos resultados obtidos, o autor inferiu que a combinação das técnicas Repetitive Transcranial Magnetic Stimulation e Sensory Cueing é mais eficaz que a técnica isolada na redução dos sintomas da SN subaguda (Scores do BIT Pré-intervenção: $\mathrm{Gl}=59.0 \pm 35.3 ; \quad \mathrm{GI}=56.0 \pm 32.2$; $\mathrm{GC}=58.4 \pm 31.0$ / Pós intervenção: $\mathrm{Gl1}=99.6 \pm 33.0$; $\mathrm{GI}=88.2 \pm 28.7 ; \mathrm{GC}=72.7 \pm 33.1$ ). Contudo, não se observou nenhum resultado significativo no aumento da independência nas AVDs (Scores da CBS Préintervenção: $\mathrm{GI}=18.5 \pm 6.8 ; \mathrm{GI}=21.2 \pm 6.5 ; \mathrm{GC}=20.5 \pm 5.8$ / Pós intervenção: $G|1=14.1 \pm 7.0 ; \quad G| 2=16.4 \pm 5.8$; $\mathrm{GC}=17.9 \pm 6.5)$ (Tabela 2).

Relativamente à dose de intervenção, os estudos realizaram 1 sessão diária, 2 a 5 vezes por semana ao longo de 1 a 4 semanas. Por fim, no que se refere ao follow-up, 2 estudos $24, \underline{26}$ realizaram-no de forma pouco clara, visto que um deles ${ }^{26}$ não especificou os motivos da desistência de alguns participantes e o outro 24 não realizou uma análise detalhada dos resultados. 


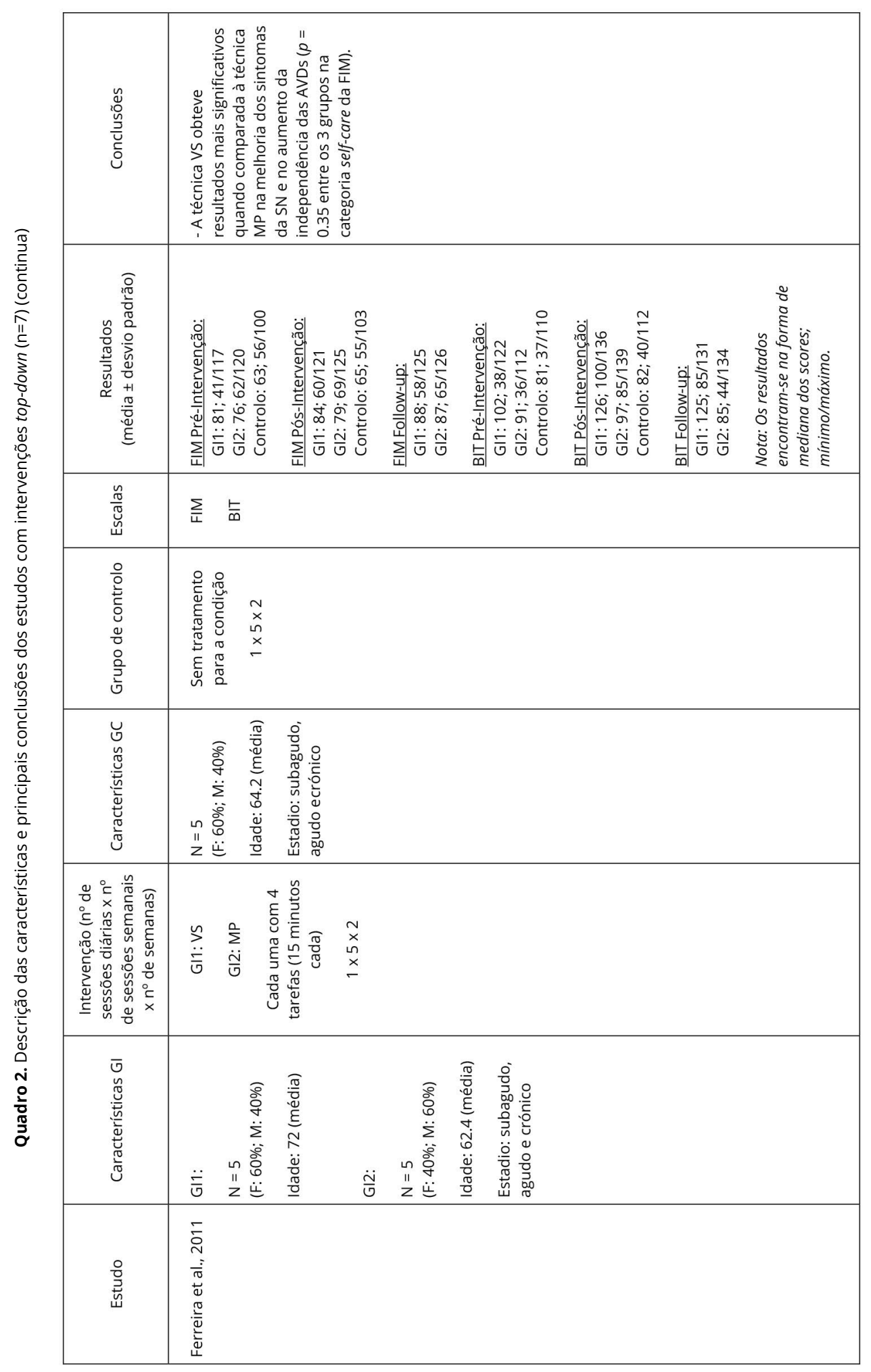




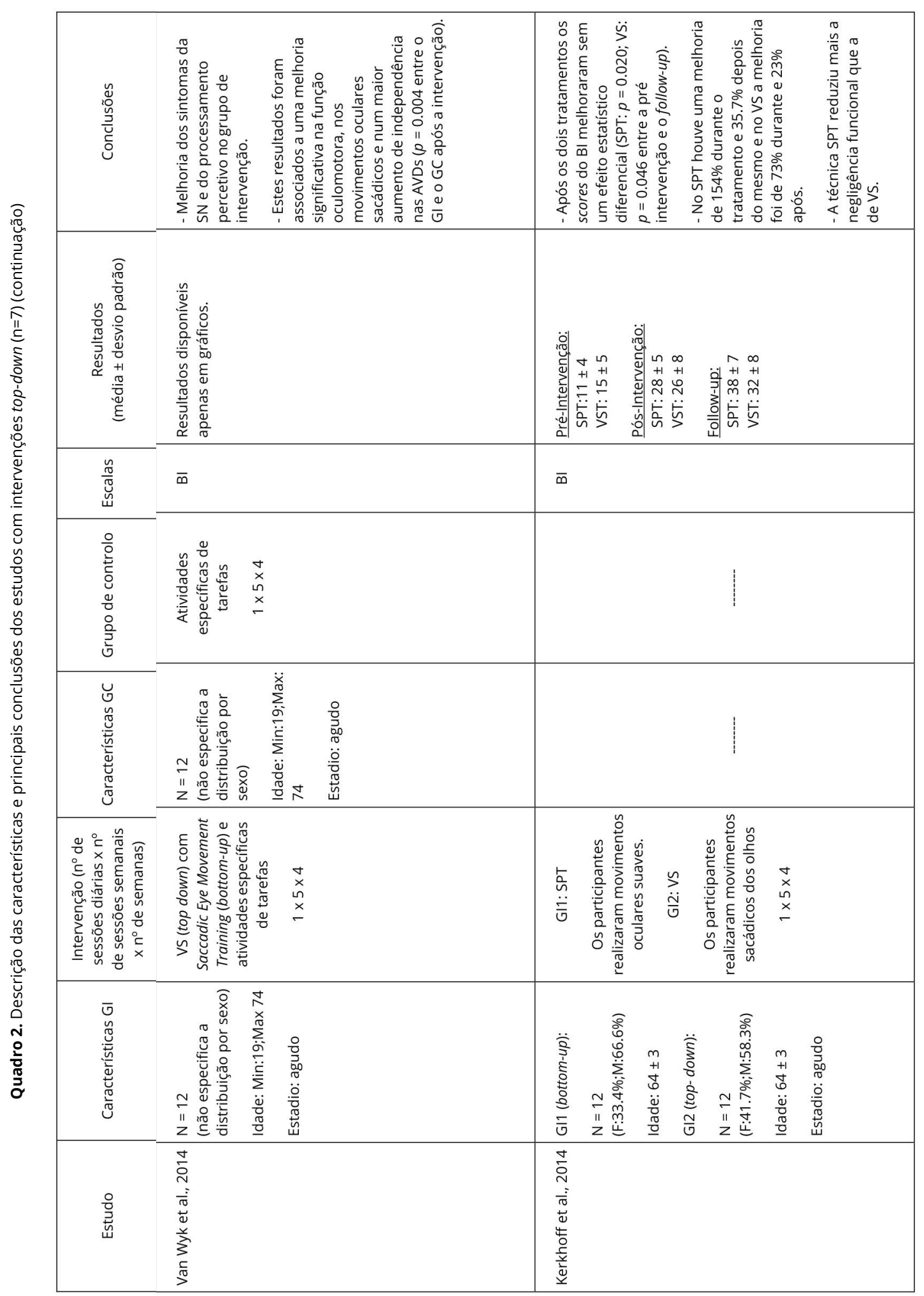




\begin{tabular}{|c|c|c|c|c|c|}
\hline 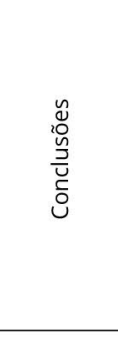 & 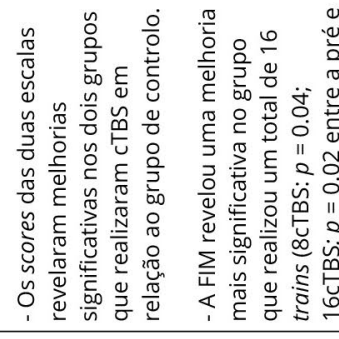 & 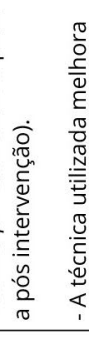 & 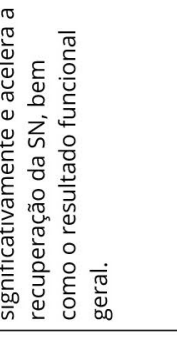 & 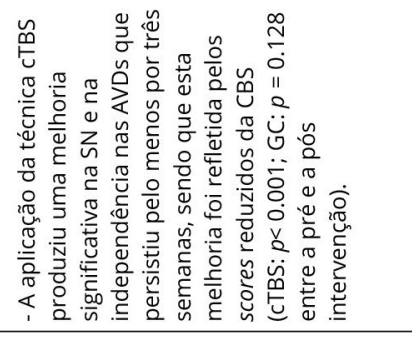 & \\
\hline 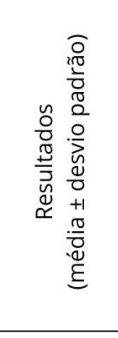 & 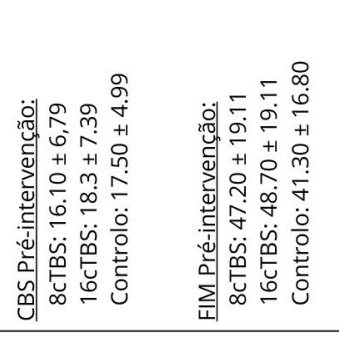 & 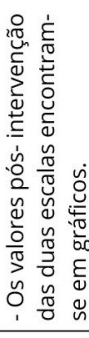 & & 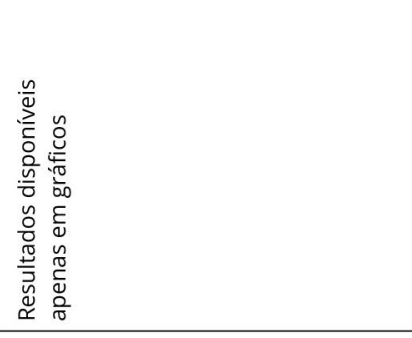 & \\
\hline $\begin{array}{l}\frac{n}{\tilde{D}} \\
\frac{\tilde{J}}{\tilde{H}}\end{array}$ & $\begin{array}{l}\sum_{i} \\
\tilde{u} \\
\underline{u}\end{array}$ & & & $\stackrel{\tilde{U}}{\tilde{U}}$ & \\
\hline 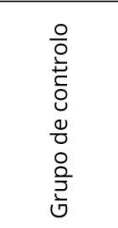 & 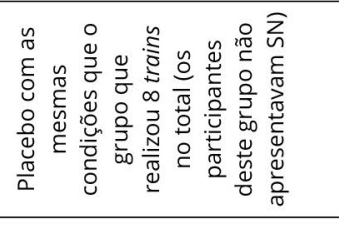 & & & 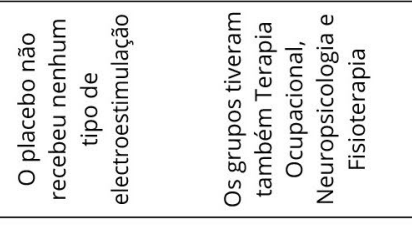 & \\
\hline 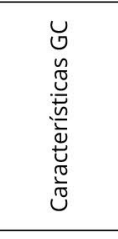 & 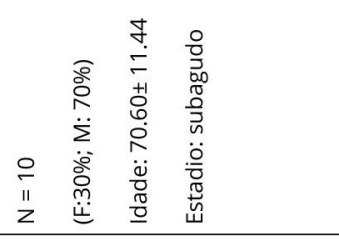 & & & 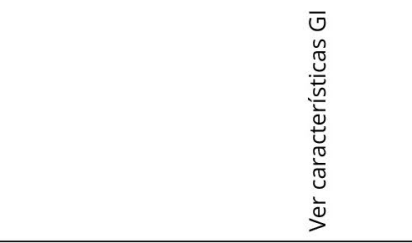 & \\
\hline 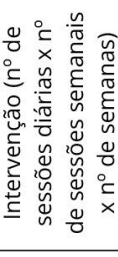 & 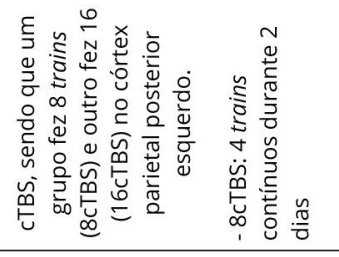 & 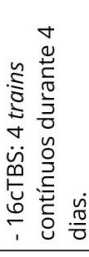 & 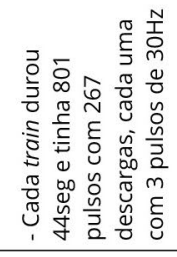 & 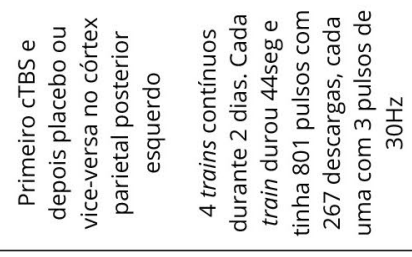 & 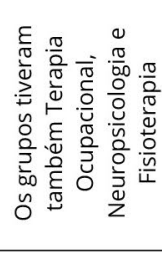 \\
\hline 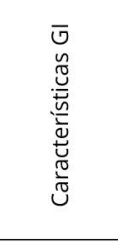 & 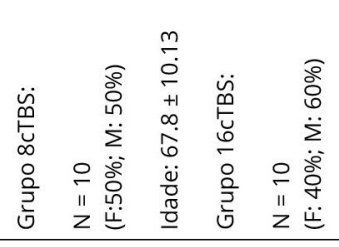 & 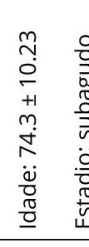 & & 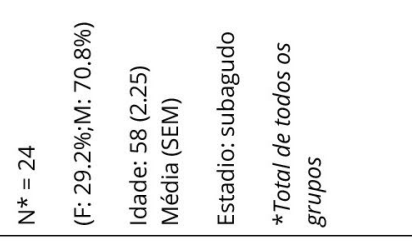 & \\
\hline $\begin{array}{l}\text { 율 } \\
\text { 离 }\end{array}$ & 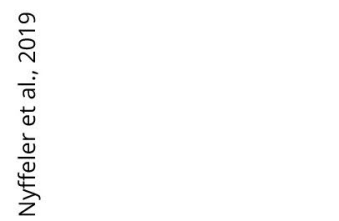 & & & 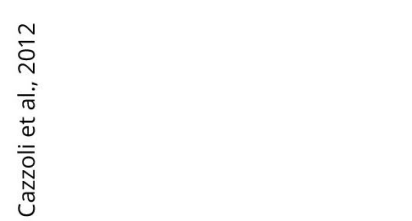 & \\
\hline
\end{tabular}




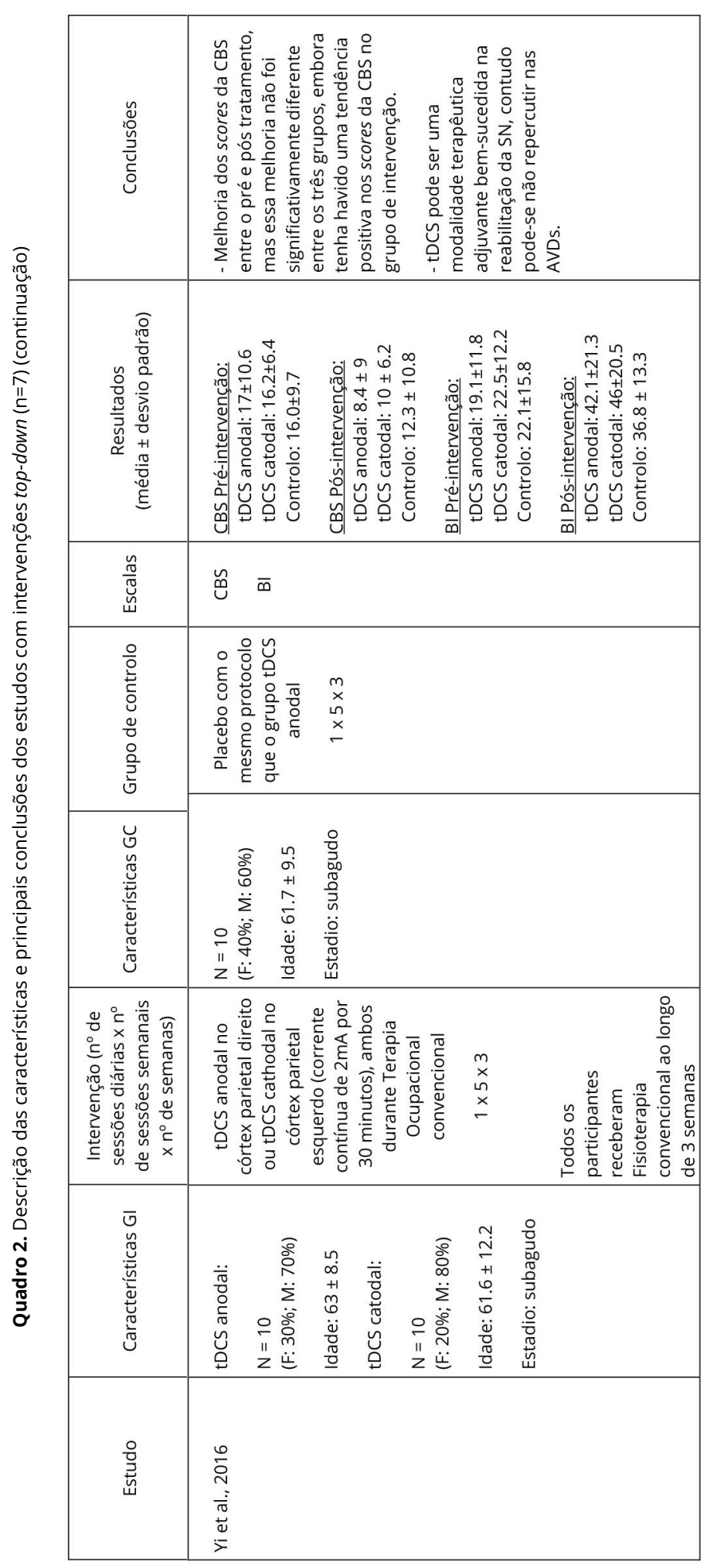




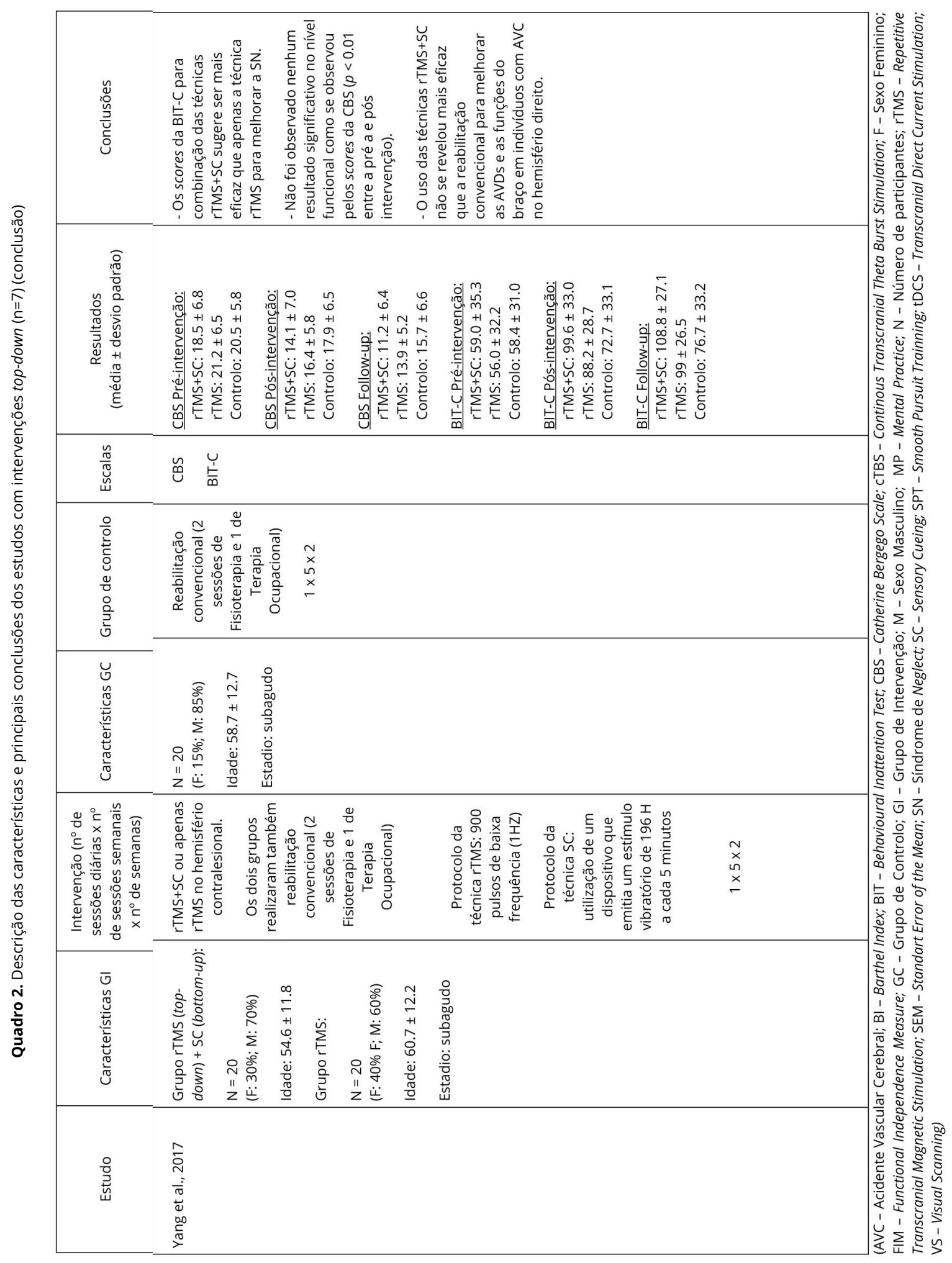


A avaliação do risco de viés dos estudos foi realizada utilizando a Joanna Briggs Institute Critical Appraisal Checklist for RCTs (Quadro 3). Com base na soma dos itens com resposta positiva, 3 dos artigos com intervenções bottom-up $2 \frac{18,20,21}{2}$ apresentam um nível de qualidade moderada, 11 artigos com intervenções bottom-up ou top-down 11,13-15,17,19,22,24,26,27 um nível elevado e 2 estudos com intervenções top-down ${ }^{16,23}$ apresentam um nível excelente.

As principais falhas na realização dos estudos estão nas questões " 5 - A intervenção foi aplicada de forma cega?"14,15,18,22,24,25, “6 - Todos os avaliadores que mediram o resultado-chave, fizeram-no de forma cega?" $15,18-22 \mathrm{e}$ "11 - Os resultados foram medidos de forma confiável?"1,13,25-27,16-18,20-24. As falhas identificadas nestas questões podem gerar vieses de observação e informação, uma vez que o conhecimento acerca do grupo a pertence o participante, pode enviesar a atuação do terapeuta (questão 5) e/ou avaliador (questão 6). Além disso, considera-se que uma medição foi feita de forma confiável (questão 11) quando é reportada informação acerca do número de avaliadores, treino dos avaliadores, a confiabilidade intra-examinador e inter-examinadores. Este requisito apenas foi claramente descrito num estudo (14); os restantes estudos não forneceram informação que permitisse a sua avaliação. 


\begin{tabular}{|c|c|c|c|c|c|c|c|c|}
\hline 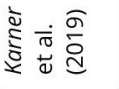 & + & + & + & + & + & n. & & ' \\
\hline 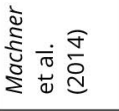 & + & + & + & + & r. & n. & + & + \\
\hline 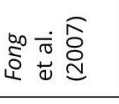 & + & + & + & + & , & + & + & + \\
\hline 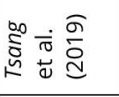 & + & + & + & + & + & + & + & ' \\
\hline 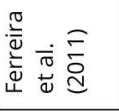 & + & n. & + & n. & + & + & + & + \\
\hline 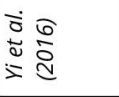 & + & + & + & + & r. & + & + & + \\
\hline 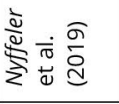 & + & + & + & + & + & + & + & r. \\
\hline 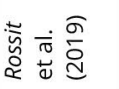 & + & + & + & + & . & , & + & + \\
\hline 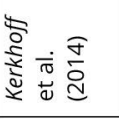 & + & + & + & + & + & + & + & + \\
\hline 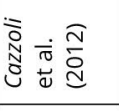 & + & + & + & + & + & + & + & + \\
\hline 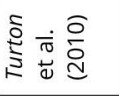 & + & + & + & + & . & . & + & . \\
\hline 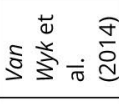 & + & + & + & + & r. & + & + & r. \\
\hline 旁㐫远递 & + & + & + & + & + & n. & + & + \\
\hline 苂 & + & ' & + & + & . & + & + & . \\
\hline 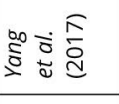 & + & + & + & + & + & + & . & + \\
\hline 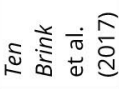 & + & + & + & + & + & ' & . & ' \\
\hline 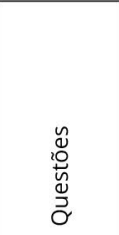 & 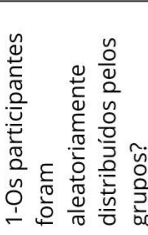 & 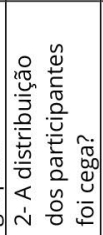 & 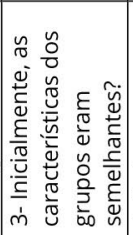 & 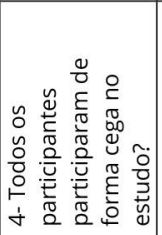 & 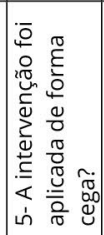 & 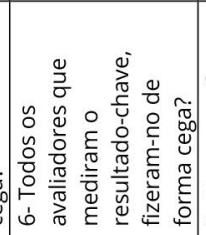 & 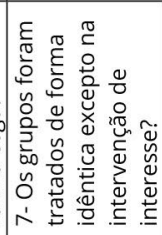 & 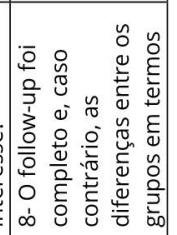 \\
\hline
\end{tabular}




\begin{tabular}{|c|c|c|c|c|c|c|c|c|}
\hline 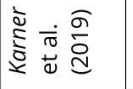 & & + & + & r. & , & & + & $\sum_{\infty}^{m}$ \\
\hline 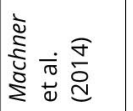 & & + & + & r. & + & & + & 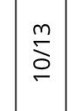 \\
\hline 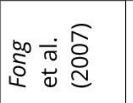 & & + & + & r. & + & & + & $\stackrel{m}{\equiv}$ \\
\hline 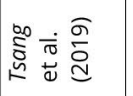 & & + & + & n. & + & & + & $\stackrel{m}{\equiv}$ \\
\hline 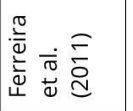 & & + & + & n. & , & & + & $\sum_{\sigma}^{m}$ \\
\hline 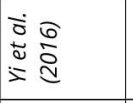 & & + & + & n. & ' & & + & $\sum_{0}^{m}$ \\
\hline 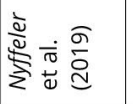 & & + & + & r. & + & & + & $\stackrel{m}{\equiv}$ \\
\hline 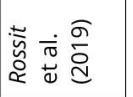 & & + & + & 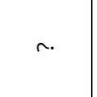 & + & & + & $\stackrel{m}{\grave{c}}$ \\
\hline 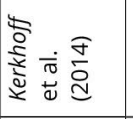 & & + & + & r. & + & & + & $\stackrel{m}{\stackrel{m}{\sim}}$ \\
\hline 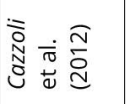 & & + & + & n. & + & & + & $\stackrel{m}{\stackrel{m}{c}}$ \\
\hline 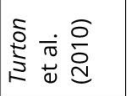 & & + & + & r. & , & & + & $\frac{m}{\infty}$ \\
\hline 离 & & + & + & r. & r. & & + & $\frac{m}{\sigma}$ \\
\hline 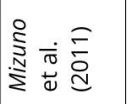 & & + & + & n. & + & & + & $\stackrel{m}{\equiv}$ \\
\hline 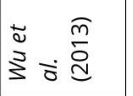 & & + & + & + & + & & + & $\sum_{0}^{m}$ \\
\hline 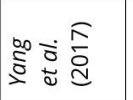 & & + & + & r. & + & & + & $\stackrel{m}{=}$ \\
\hline 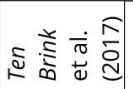 & & + & + & n. & n. & & + & $\sum_{\infty}^{m}$ \\
\hline 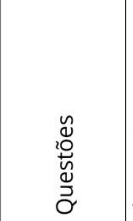 & 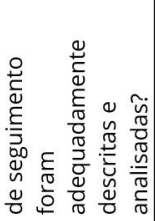 & 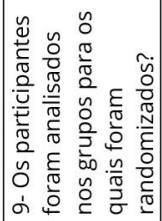 & 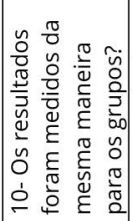 & 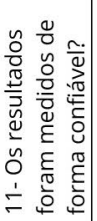 & 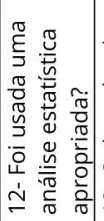 & 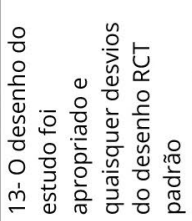 & 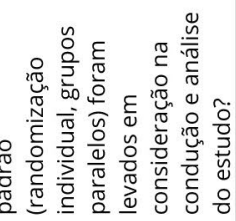 & 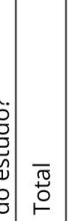 \\
\hline
\end{tabular}




\section{Discussão}

Esta revisão sistemática teve como objetivo caracterizar as abordagens (bottom-up ou top-down) com maior impacto nas AVDs em adultos e idosos com SN. Foram incluídos 16 estudos, dos quais 9 contêm técnicas da abordagem bottom-up, 6 contêm técnicas da abordagem top-down e 1 estudo compara os efeitos das diferentes abordagens ${ }^{16}$. A presente revisão sistemática parece ajudar a esclarecer que (a) diferentes técnicas nas abordagens bottom-up e top-down revelaram ser eficazes no aumento da independência das AVD's; (b) uma melhoria dos sintomas da SN com a implementação destas estratégias não se traduz numa melhoria na independência nas AVDs; (c) a classificação em "bottom-up" e "top-down" parece ter na sua base alguns princípios difíceis de explicar; (d) a escolha destas abordagens pode estar dependente do nível cognitivo do paciente.

Na abordagem bottom-up, as técnicas que se mostraram eficazes no aumento da independência nas AVDs foram Visuomotor Feedback Training22, Smooth Pursuit Eye Movement Training ${ }^{16}$ e Eye Patching quando coadjuvada com Constraint-induced Therapy ${ }^{14}$. Por sua vez, na abordagem top-down as técnicas que se revelaram eficazes no aumento da independência das AVDs foram Visual Scanning 16,24,27, Mental Practice $^{16}$, Continuous Theta Burst Stimulation $\underset{23,26}{ }$ e Transcranial Direct Current Stimulation ${ }^{25}$, quer realizadas de forma isolada ou combinadas com outras intervenções.

Na técnica Prism Adaptation ${ }^{18-20}$ os resultados foram controversos quanto ao aumento da independência nas AVDs. Assim, apenas o estudo de Mizuno et al. $(2011)^{19}$ relatou uma melhoria na independência das AVDs em participantes com SN subaguda, pelo que utilizou uma dose de intervenção maior (2 sessões diárias durante 5 dias por semana ao longo de 2 semanas) do que os restantes estudos. Deste modo, uma maior dose de intervenção (por exemplo, um maior número de sessões diárias) para esta técnica parece traduzir-se num efeito mais significativo ao nível da independência nas AVDs, uma vez que os estudos que realizaram apenas 1 sessão diária não relataram efeitos positivos ao nível das AVDs ${ }^{18-19}$.
$\mathrm{Na}$ análise dos estudos foi igualmente evidente que uma melhoria dos sintomas da SN não se traduz numa melhoria na independência nas AVDs. Tal elação observou-se no estudo de Turton et al. (2010) que avaliou a técnica Prism Adaptation ${ }^{18}$ (score CBS: Pré intervenção: grupo de intervenção $=12 \pm 5$ grupo de controlo $=$ $12 \pm 4$; Pós intervenção: grupo de intervenção = variação positiva de $3.5 \pm 3.1$; grupo de controlo = variação positiva de $3.3 \pm 2.5$ ) (score BIT-C: Pré intervenção: grupo de intervenção $=88 \pm 71$; grupo de controlo $=109 \pm$ 70; Pós intervenção: grupo de intervenção = variação positiva de $14.8 \pm 18.8$; grupo de controlo = variação positiva de $9.7 \pm 15.9$ ), no estudo de Tsang et al. (2009) que aplicou a técnica Eye Patching de forma isolada ${ }^{17}$ (score FIM: Pré intervenção: grupo de intervenção = $56.24 \pm 15.72$ grupo de controlo $=46.94 \pm 16.15$; Pós intervenção: grupo de intervenção $=16.00 \pm 14.24$; grupo de controlo $=12.41 \pm 14.21$ ) (score BIT: Pré intervenção: grupo de intervenção = $34.84 \pm 25.99$; grupo de controlo $=43.94 \pm 34.56$; Pós intervenção: grupo de intervenção $=25.06 \pm 30.81$; grupo de controlo $=8.29$ \pm 10.35 ) e no estudo de Yang et al. (2017) que utilizou a técnica Repetitive Transcranial Magnetic Stimulation (topdown) de forma combinada (grupo 1) com a técnica Sensory Cueing (bottom-up) e isolada (grupo 2) (1) (score CBS: Pré intervenção: grupo $1=18.5 \pm 6.8$; grupo $2=21.2 \pm 6.5$; controlo $=20.5 \pm 5.8$; Pós intervenção: grupo 1 = $14.1 \pm 7.0$; grupo 2 = $16.4 \pm 5.8$; controlo = $17.9 \pm 6.5$ ) (score de BIT- C: Pré intervenção: grupo 1 $=59.0 \pm 35.3$; grupo $2=56.0 \pm 32.2$; controlo $=58.4 \pm$ 31.0; Pós intervenção: grupo $1=99.6 \pm 33.0$; grupo $2=$ $88.2 \pm 28.7$; controlo $=72.7 \pm 33.1$ )

Para além do exposto, a classificação das técnicas nestas duas abordagens (bottom-up e top-down) parece ter princípios difíceis de explicar. $\mathrm{Na}$ abordagem bottom-up a variedade de mecanismos neurofisiológicos inerentes às técnicas é consideravelmente maior. Por exemplo, a técnica Prism Adaptation ${ }^{18,20}$, tal como a técnica Eye Patching ${ }^{14}$ envolve uma mudança do campo visual, mas também integra um julgamento percetivo visual como a técnica Visuomotor Feedback Training 22 . Adicionalmente, o robot PARO pertence à categoria de robots de estimulação interativa projetados especificamente para fins terapêuticos e no es-

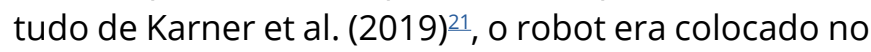
lado negligenciado do participante. Quanto à técnica Smooth Pursuit Eye Movement Training, esta corresponde a movimentos oculares usados para estabilizar a imagem de um objeto em movimento na fóvea ${ }^{16}$. 
Por sua vez, ao contrário da abordagem bottom-up, na abordagem top-down é possível agrupar as suas técnicas de acordo com os seus mecanismos neurofisiológicos. Deste modo, a técnica Visual Scanning envolve o reconhecimento visual dado que é inspirada nas técnicas de modificação de comportamento e consciencializa o indivíduo sobre a presença de estímulos no lado contralesional, interferindo nos níveis de atenção e no processamento da informação ${ }^{29}$. A técnica de Visual Scanning recorre tanto às aprendizagens previamente adquiridas como também à consciência da condição, o mesmo não acontecendo com as técnicas Optokinetic Stimulation e Smooth Pursuit Eye Movement Training, que não necessitam de altos níveis de consciência acerca da sua condição por parte do indivíduo. Para além do mais, a técnica Mental Practice consiste em realizar mentalmente as diferentes etapas de uma ação/tarefa requerendo de igual forma elevados níveis cognitivos ${ }^{27}$. Quanto às outras técnicas incluídas na abordagem top-down, ou seja, Continuos Theta Burst Stimulation ${ }^{23}$, Transcranial Direct Current Stimulation $\frac{25}{5}$ e Repetitive Transcranial Magnetic Stimulation ${ }^{1}$, estas podem ser integradas em técnicas de electroestimulação que têm por base o princípio de inter-rivalidade hemisférica, podendo atuar ao nível da neuromodelação quer por excitação do lado lesionado quer por inibição do lado contralesional1,23,25.

O processo de escolha das abordagens na reabilitação da SN pode também estar condicionado pelo perfil cognitivo necessário à sua implementação. Por exemplo, na abordagem top-down existem técnicas que envolvem níveis de participação ativa e níveis cognitivos distintos entre si como a técnica Visual Scanning e as técnicas de electroestimulação. A primeira solicita níveis cognitivos mais elevados e níveis ativos de participação por parte do indivíduo, uma vez que requer uma colaboração do indivíduo e um reconhecimento visual do mesmo 29 .

Apesar da classificação em abordagens bottomup e top-down, esta distribuição parece ainda ser contraditória para algumas técnicas em específico, como acontece com a técnica Repetitive Transcranial Magnetic Stimulation ${ }^{1}$. Segundo o autor do estudo incluído nesta RS, Yang et al. (2017)1', e o autor Carmelo $(2015)^{36}$ a técnica mencionada pertence à abordagem top-down, contudo Dintén-Fernández et al. (2019) ${ }^{37}$ insere-a na abordagem bottom-up. Esta dificuldade pode dever-se ao facto da técnica exigir baixos níveis de participação ativa por parte do indivíduo, mas levar a modificações no processamento cerebral sensoriomotor ou cognitivo ${ }^{1}$.

\section{Conclusão}

Desta revisão sistemática não é possível concluir qual a abordagem (bottom-up e top-down) que apresenta um maior impacto no aumento da independência das AVD's, uma vez que ambas incluem técnicas que revelam resultados positivos. Os princípios que estão na base da classificação das técnicas estão relacionados com os mecanismos neurofisiológicos, muito distintos entre si e de caraterização ambígua entre autores, dificultando a escolha clínica das abordagens.

\section{Contribuições das autoras}

Martins AR, Vieira A, Oliveira C, Bártolo M participaram na conceção, delineamento, pesquisa, análise e interpretação dos resultados e redação do artigo científico. Silva CG participou no delineamento, pesquisa, análise e redação do artigo científico. Rosa M participou na análise e interpretação dos resultados e redação do artigo científico bem como, na orientação da elaboração do artigo.

\section{Conflitos de interesses}

Nenhum conflito financeiro, legal ou político envolvendo terceiros (governo, empresas e fundações privadas, etc.) foi declarado para nenhum aspecto do trabalho submetido (incluindo, mas não se limitando a subvenções e financiamentos, participação em conselho consultivo, desenho de estudo, preparação de manuscrito, análise estatística, etc.).

\section{Agradecimentos}

As autoras agradecem ao Instituto Politécnico de Leiria pelo apoio na conceção, desenho, planeamento e execução deste estudo.

\section{Referências}

1. Yang NY, Fong KN, Li-Tsang CW, Zhou D. Effects of repetitive transcranial magnetic stimulation combined with sensory cueing on unilateral neglect in subacute patients with right hemispheric stroke: A randomized controlled study. Clin Rehabil. 2017;31(9):1154-63. doi: 10.1177/0269215516679712 
2. Conti RP, Arnone JM. Unilateral Neglect: Assessment and Rehabilitation. Int J Neurosci Behav Sci. 2016;4(1):1-10. doi: 10.13189/ijnbs.2016.040101

3. Lisa LP, Jughters A, Kerckhofs $E$. The effectiveness of different treatment modalities for the rehabilitation of unilateral neglect in stroke patients: a systematic review. NeuroRehabilitation. 2013;33(4):611-20. doi: 10.3233/NRE-130986

4. Li K, Malhotra PA. Spatial neglect. Practical Neurology. 2015;15:333-9. doi: 10.1136/practneurol-2015-001115

5. Molenberghs $P$, Sale M V, Mattingley JB. Is there a critical lesion site for unilateral spatial neglect? A meta-analysis using activation likelihood estimation. Front Hum Neurosci. 2012;6:78. doi: $\underline{10.3389 / \text { fnhum. } 2012.00078}$

6. Varalta V, Munari D, Pertile L, Fonte C, Vallies G, Chemello E et al. Effects of neck taping in the treatment of hemispatial neglect in chronic stroke patients: A pilot, single blind, randomized controlled trial. Medicina. 2019;55(4):108. doi: 10.3390/ medicina55040108

7. Azouvi $P$, Jacquin-Courtois $S$, Luauté J. Rehabilitation of unilateral neglect: Evidence-based medicine. Ann Phys Rehabil Med. 2017;60(3):191-197. doi: 10.1016/j.rehab.2016.10.006

8. Marshall RS. Rehabilitation approaches to hemineglect. Neurologist. 2009;15(4):185-92. doi: $10.1097 /$

NRL.0b013e3181942894

9. Liu KPY, Hanly J, Fahey P, Fong SSM, Bye R. A Systematic Review and Meta-Analysis of Rehabilitative Interventions for Unilateral Spatial Neglect and Hemianopia Poststroke From 2006 Through 2016. Arch Phys Med Rehabil. 2019;100(5):956-979. doi: 10.1016/j. apmr.2018.05.037

10. Stewart LA, Clarke M, Rovers M, Riley RD, Simmonds M, Stewart $G$ et al. Preferred reporting items for a systematic review and meta-analysis of individual participant data: The PRISMAIPD statement. JAMA. 2015;313(16):1657-65. doi: $10.1001 /$ jama.2015.3656

11. Tufanaru C, Munn Z, Aromataris E, Campbell J, Hopp L. Joanna Briggs Institute Reviewer's Manual. Adelaide, Australia: Joanna Briggs Institute; 2017.

12. McHugh ML. Interrater reliability: The kappa statistic. Biochem Med. 2012;22(3):276-82.

13. Fong KNK, Chan MKL, Ng PPK, Tsang MHM, Chow KKY, Lau $C W L$ et al. The effect of voluntary trunk rotation and half-field eye-patching for patients with unilateral neglect in stroke: $\mathrm{A}$ randomized controlled trial. Clin Rehabil. 2007;21(8):729-41. doi: $\underline{10.1177 / 0269215507076391}$
14. Wu CY, Wang TN, Chen YT, Lin KC, Chen YA, Li HT et al. Effects of constraint-induced therapy combined with eye patching on functional outcomes and movement kinematics in poststroke neglect. Am J Occup Ther. 2013;67(2):236-45.

15. Machner B, Könemund I, Sprenger A, Von Der Gablentz J, Helmchen C. Randomized controlled trial on Hemifield eye patching and Optokinetic stimulation in acute spatial neglect. Stroke. 2014;45(8):2465-8. doi: 10.1161/STROKEAHA.114.006059

16. Kerkhoff G, Bucher L, Brasse M, Leonhart E, Holzgraefe M, Völzke $V$ et al. Smooth pursuit "bedside" training reduces disability and unawareness during the activities of daily living in Neglect: A randomized controlled trial. Neurorehabil Neural Repair. 2014;28(6):554-63. doi: 10.1177/1545968313517757

17. Tsang MHM, Sze KH, Fong KNK. Occupational therapy treatment with right half-field eye-patching for patients with subacute stroke and unilateral neglect: A randomised controlled trial. Disabil Rehabil. 2009;31(8):630-7. doi: $10.1080 / 09638280802240621$

18. Turton AJ, O'Leary K, Gabb J, Woodward R, Gilchrist ID. A single blinded randomised controlled pilot trial of prism adaptation for improving self-care in stroke patients with neglect. Neuropsychol Rehabil. 2010;20(2):180-96. doi: 10.1080/09602010903040683

19. Mizuno K, Tsuji T, Takebayashi T, Fujiwara T, Hase K, Liu M. Prism adaptation therapy enhances rehabilitation of stroke patients with unilateral spatial neglect: A randomized, controlled trial. Neurorehabil Neural Repair. 2011;25(8):711-20. doi: $10.1177 / 1545968311407516$

20. Brink AFT, Visser-Meily JMA, Schut MJ, Kouwenhoven M, Eijsackers ALH, Nijboer TCW. Prism Adaptation in Rehabilitation? No Additional Effects of Prism Adaptation on Neglect Recovery in the Subacute Phase Poststroke: A Randomized Controlled Trial. Neurorehabil Neural Repair. 2017;31(12):1017-28. doi: $\underline{10.1177 / 1545968317744277}$

21. Karner S, Stenner H, Spate M, Behrens J, Krakow K. Effects of a robot intervention on visuospatial hemineglect in postacute stroke patients: a randomized controlled trial. Clin Rehabil. 2019;33(12):1940-8. doi: 10.1177/0269215519865993

22. Rossit S, Benwell CSY, Szymanek L, Learmonth G, McKernanWard L, Corrigan E et al. Efficacy of home-based visuomotor feedback training in stroke patients with chronic hemispatial neglect. Neuropsychol Rehabil. 2019;29(2):251-72. doi: 10.1080/09602011.2016.1273119

23. Cazzoli D, Müri RM, Schumacher R, von Arx S, Chaves S, Gutbrod K et al. Theta Burst Stimulation Reduces Disability During the Activities of Daily Living in Spatial Neglect. 2012;135(Pt 11):3426-39. doi: 10.1093/brain/aws182 
24. Van Wyk A, Eksteen CA, Rheeder P. The effect of visual scanning exercises integrated into physiotherapy in patients with unilateral spatial neglect poststroke: A matched-pair randomized control trial. Neurorehabil Neural Repair. 2014;28(9):856-73. doi: $10.1177 / 1545968314526306$

25. Yi YG, Chun MH, Do KH, Sung EJ, Kwon YG, Kim DY. The effect of transcranial direct current stimulation on neglect syndrome in stroke patients. Ann Rehabil Med. 2016;40(2):223-9. doi: 10.5535/ arm.2016.40.2.223

26. Nyffeler T, Vanbellingen T, Kaufmann BC, Pflugshaupt T, Bauer D, Frey J et al. Theta burst stimulation in neglect after stroke: functional outcome and response variability origins. Brain. 2019;142(4):992-1008. doi: 10.1093/brain/awz029

27. Ferreira HP, Lopes MAL, Luiz RR, Cardoso L, André C. Is visual scanning better than mental practice in hemispatial neglect? Results from a pilot study. Top Stroke Rehabil. 2011;18(2):155-61. doi: $10.1310 /$ tsr1802-155

28. Bedford F. Prism adaptation. Psychol Learn Motiv. 1993.

29. Priftis K, Passarini L, Pilosio C, Meneghello F, Pitteri M. Visual Scanning Training, Limb Activation Treatment, and prism adaptation for rehabilitating left neglect: Who is the winner? Front Hum Neurosci. 2013;7:360. doi: 10.3389/fnhum.2013.00360

30. Dundon NM, Bertini C, Làdavas E, Sabel BA, Gall C. Visual rehabilitation: Visual scanning, multisensory stimulation and vision restoration trainings. Front Behav Neurosci. 2015;9:192. doi: 10.3389/fnbeh.2015.00192
31. Fu W, Song $W$, Zhang $Y$, Yang $Y$, Huo S, Zhang R et al. Long-term effects of continuous theta-burst stimulation in visuospatial neglect. J Int Med Res. 2015;43(2):196-203. doi: $\underline{10.1177 / 0300060513498663}$

32. Niehorster DC, Siu WWF, Li L. Manual tracking enhances smooth pursuit eye movements. J Vis. 2015;15(15):11. doi: 10.1167/15.15.11

33. Rahnev D, Kok P, Munneke M, Bahdo L, Lange FP, Lau H. Continuous theta burst transcranial magnetic stimulation reduces resting state connectivity between visual areas. J Neurophysiol. 2013;110(8):1811-21. doi: 10.1152/jn.00209.2013

34. Thair H, Holloway AL, Newport R, Smith AD. Transcranial direct current stimulation (tDCS): A Beginner's guide for design and implementation. Front Neurosci. 2017;11:641. doi: 10.3389/ fnins.2017.00641

35. Mishra BR, Sarkar S, Praharaj SK, Mehta VS, Diwedi S, Nizamie $\mathrm{SH}$. Repetitive transcranial magnetic stimulation in psychiatry. Ann Indian Acad Neurol. 2011;14(4):245-251. doi: 10.4103/09722327.91935

36. Carmelo C. Bottom-Up or Top-Down Approach? Understanding the Way to Reach the Milestone of Recovery in Stroke. Int J Neurorehabilitation. 2015;2(2). doi: 10.4172/2376$\underline{0281.1000 \mathrm{e} 107}$

37. Dintén-Fernández A, Fernández-González P, Koutsou A, Alguacil-Diego IM, Laguarta-Val S, Molina-Rueda F. Enfoques top-down y bottom-up para el tratamiento de la heminegligencia espacial en sujetos con ictus: revisión sistemática. Rehabilitación. 2019;53(2):93-103. doi: 10.1016/j.rh.2018.10.001 